\title{
Automatic hybrid RANS/LES strategy for industrial CFD
}

\author{
Grégoire Pont, Paola Cinnella, J.C. Robinet, and Pierre Brenner
}

\begin{abstract}
An automatic HRL ${ }^{1}$ strategy is investigated in FLUSEPA, a finite-volume solver developed by Airbus Defense \& Space. A HRL turbulence model is coupled to a high-order hybrid numerical approximation method. Concerning the turbulence model, the well-known $k-\varepsilon$ two equations RANS turbulence model is sensitized to the grid as suggested by Perot and Gadebusch [1]. Concerning the numerical strategy, a third-order accurate upwind approximation method is locally re-centered in vortex dominated regions to achieve non-dissipative fourth-order accuracy. Results are presented for a 2D backward facing step and an an axisymmetry backward facing step, which represent good prototypes of after body flows.
\end{abstract}

\section{Introduction}

There are three kinds of viscosity in a numerical simulation of turbulent flow : laminar viscosity $v$, eddy viscosity $v_{t}$ introduced by RANS (Boussinesq hypothesis) or SGS model in use, and numerical viscosity $v_{n}$ intrinsic to the numerical scheme. The second kind of viscosity is equal to zero for so called "implicit"modelling approaches (see e.g Ref[2]). The main idea of a hybrid turbulence model is to reduce the turbulent viscosity $v_{t}$ to locally solve unsteady turbulent structures. In the resolved part of the turbulent spectrum, the numerical viscosity must be negligible compared to eddy viscosity, otherwise, turbulent structures will be dissipated by the numerical scheme.

Grgoire Pont,

Airbus Space and Defense / Dynfluid Laboratory, e-mail: gregoire.pont@ensam.eu

Paola Cinella $\cdot$ J.C. Robinet

Dynfluid Laboratory,

Pierre Brenner

Airbus Space and Defense, Les Mureaux, France

${ }^{1}$ Hybrid RANS/LES 
In this work, the numerical solver in use is FLUSEPA, the unstructured finitevolume solver developed by Airbus Defense \& Space company to calculate compressible, multidimensional, unsteady, viscous and reactive flow over bodies in relative motion. The numerical scheme used in this solver is designed for highly compressible flows and has good shock capturing capabilities. The solver is based on a high order Godunov type method along with a MUSCL-like reconstruction. All the required derivatives are calculated by a successive corrections algorithm. The Godunov methods are well known to be suitable for compressible flows with shocks because they introduce a numerical dissipation that damps non-physical oscillations and ensures the stability of the method, but they are much too dissipative for HRL calculations. In the quest for a compromise between computational cost and resovability properties of the numerical method, we retain a hybrid scheme, combining a 3rd-order accurate version of the present scheme in inviscid regions with a fourthorder non dissipative scheme in regions dominated by turbulent flow structures.

\section{Governing equations}

We look for the numerical solutions of the compressible Navier-Stokes equations within Reynolds-Averaged form or in filtered form, and supplemented by a turbulence or subgrid model, respectively. In the following, the Reynolds/subgrid stress tensor is described by using an eddy viscosity model, and is supposed to be related to the average/filtered velocity gradient via an eddy viscosity coefficient $v_{t}$. To compute $v_{t}$, automatics HRL models are considered based on a modification of well known RANS models. Precisely, in this work we consider the hybrid $k-\varepsilon$ model developed by Perot and Gadebusch [1]. Grid sensitization of the underlying RANS models is achieved by introducing an energy transfer parameter $\alpha$ :

$$
\alpha=1.5\left(1-C^{*}\left(\frac{k}{k+k_{r}}\right)^{2}\left[\left(\frac{\Delta x_{i}}{\sqrt{k_{r}}} \frac{\partial \sqrt{k_{r}}}{\partial x_{i}}\right)^{2}+0.11\right]^{-1}\right)
$$

where $k_{r}$ is the resolved turbulent kinetic energy, $k$ the modelled kinetic energy and $C^{*}=0.28$. The $\alpha$ parameter, regions, turns the baseline two equations models into a subgrid model and keep a classical $k-\varepsilon$ model in under-resolved regions. The $\alpha$ parameter acts through two mechanisms: it pre-multiplies the Reynolds stress tensor, contributing to lower the modelled Reynolds stresses in well-resolved regions; it pre-multiplies the production term in the turbulent kinetic energy equation, contributing to lower the amount of modelled kinetic energy produced by the model and, indirectly, the eddy viscosity. The eddy viscosity formulation is also modified with respect to the standard model, by introducing a weighting factor equal to the ratio of the modelled to the total kinetic energy). Note that $\alpha$ may become negative in highly resolved regions characterized by a too large amount of modelled kinetic energy. This am- 
plifies flow instabilities by converting turbulent diffusion in antidiffusion and enables effective model transition from RANS to LES mode by enriching the frequency spectrum. The model is implemented along with a shielding function that enforces a RANS mode in attached boundary layers. Figure 1 shows a snapshot of eddy viscosity and the time-averaged distribution of modelled to total kinetic energy ratio for an axisymmetric backward facing step flow discussed in the following. The eddy viscosity is found to decrease in the shear layers and in the recirculation bubble, which allow ${ }_{i}$ the resolved kinetic energy to increase. We can see there is no resolved mbulence energy in the RANS boundary layers before the separation point.

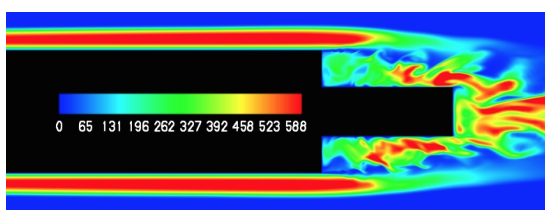

(a) Eddy viscosity ratio $v_{t} / v$

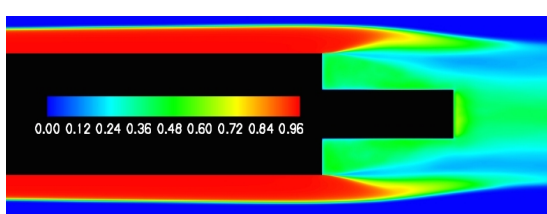

(b) Averaged modeled to total kinetic energy ratio.

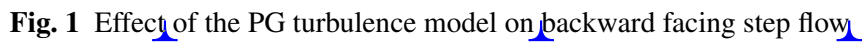

\section{Numerical method}

For the numerical approximation of system of conservation laws formed by the Navier-Stokes equations, we consider an unstructured finite volume methodology. Fluxes are integrated on each interfaces of control volume at hight order of aceuracy,

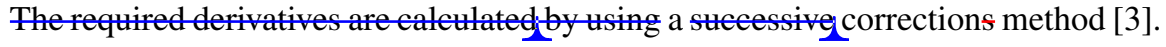
This kind of method allows to keep hight order of aecuracy $y_{\mathcal{L}}$ on general mesh-solver. The truncation error and the spectral properties of the preceding numerical scheme have been studied studied in details for a linear advection problem. Finite volume eperators with orders of ang 2 to 5 [4]. Due to the use of an upwind numerical flux at cell interfaces, all schemes in the family exhibit relatively high damping errors, which be oreduch ac euracy at the cest of a significant increase in computational complexity. Moreover, even for schemes of $4^{\text {th }}$ and $5^{\text {th }}$ accuracy, the resolvability properties of the numerical approximations are such that more than 10 points per wavelength are required to keep damping errors to within a reasonable limit. This means that, with grid resolutions typically used in industrial applications, the numerical dissipation introduced by the scheme is not compatible with a HRL simulation. In restrict our attention to the third-order scheme, which represents computational complexity, and reduce numerical dissipation in vortex-dominated regions by local re-centering of the method. Re-centering of the 
numerical fluxes leads to a fourth-order accurate, non dissipative scheme in vortexdominated regions, while keeping a 3rd-order upwind scheme in shock-dominated regions. The proposed $V C$ scheme is stable if the grid Reynolds number is below 2. Note that grid Reynolds number depends on both the laminar and the eddy viseosity in a cell. When this condition is not satisfied, the scheme is only partially centered, which comes to locally lowering the numerical dissipation coefficient. _Temporal integration is carried out by means of a second-order method, namely, the Heun scheme. Each cell advanced with nearly the its maximum allowable time step according to the CFL condition, and time consistency is ensure by sub-iterating over the cell with local time step lower than the maximum value over the domain. This time integration is well suited for unsteady problems ${ }_{2}$ small physical time scales-are needed.

\section{Backward facing step flows}

Backward facing step flows are a good prototype for after-body aerodynamics, and exhibit several unsteady phenomena. First, a Kelvin-Helmholtz vortex shedding appear at the separation point followed by a pairing process highlighted by the hot-wire techniques experiment of Troutt et al. [5] . Hairpin vortices have been observed in the recirculation bubble by Kiya and Sisaki [6]. On the other side, several researcher agree in indicating the presence of another unsteady phenomenon, called flapping motion, which is a low frequency instability. The backward facing step flow is a very good testing bench for self-adaptive HRL models because the Kelvin-Helmholtz instability is difficult to capture without delay due to the convective character of this instability and the turbulent viscosity coming from the boundary layer before the separating point.

\subsection{D backward facing step}

The test case is a geometrically 2D backward facing step with an upper wall experimentally studied by Moreau et al. [7]. The geometrical features and measures of the computational domain used in this study are detailed in figure 2 . The length before the step is chosen so as to obtain a boundary layer thickness of $0.37 \mathrm{~h}$ just before the separation point. Hereafter, we show results obtained with the PG model. Numerical simulations using ZDES modelling on a fine mesh of 3.9 million points were provided by Deck [8], which are also displayed for comparison. Details of the simulations and references are presented in the tab 1. Grids 1,2 and 3 differ only by refinement in the spanwise direction, where the number of grid point is doubled. The $y^{+}$and $x^{+}$(at the beginning of the step) for grid 1,2 and 3 is equal to 15 .

We can see the grid convergen of the model regarding the isosurface of $\lambda_{2}$ criteria colored by mean velocity-on figure 3 . Refinement in the spanwise direc- 


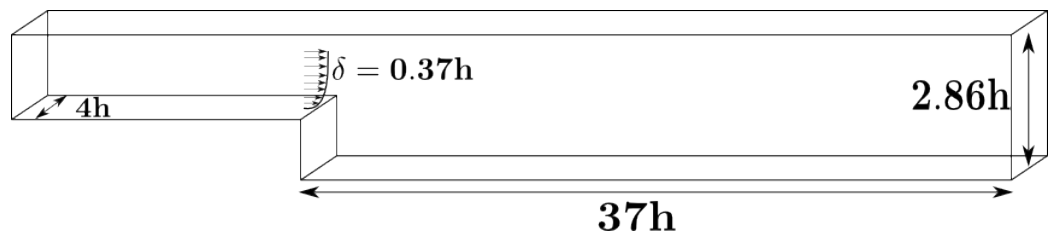

Fig. 2 Sketch of the computational domain

\begin{tabular}{|c|c|c|c|}
\hline & Present calculation & ZDES ONERA,[8] & LDV Experiment[7] \\
\hline Reynolds number & 40000 & 40000 & 40000 \\
\hline $\begin{array}{l}\text { Number } \\
\text { of } \\
\text { elements }\end{array}$ & 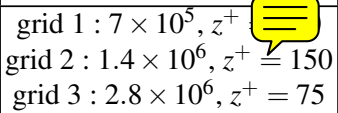 & $4.1 \sqrt{\overline{\overline{\bar{E}}}}$ & Moreau et al. \\
\hline Turbulence & Perot \& Gadebusch & ZDES & \\
\hline
\end{tabular}

Table 1 Details of calculations and experiment

tion leads to grid convergence of the model as precedingly observed by Perot and Gadebusch for isotropic decaying turbulence [1]. We can also observe the formation of hairpin vorteces in the recirculation bubble after a vortex pairing mecanism and longitudinal vortices are generated downstream of the reattachment point.

Figure 4 shows the average streamwise velocity field and streamlines, as well as velocity profiles taken at different streamwise locations in the recirculation bubble. Present results are in good agreement with the experiments. The location of the reattachment point for grid 1 and $2\left(X_{r} / H \simeq 6.2\right)$ is in good agreement witch the experiment of Hall et al. [9], of $X_{r} / H \simeq 6.8$, Driver et al. [10], $X_{r} / H \simeq 6.1$ and the direct simulation of Le et al. [11] with $X_{r} / H \simeq 6.28$. On the other hand, the shape of the secondary recirculation bubble in the corner is in good agreement with the PIV streamlines of the experiment of Hall et al. [9]. ZDES calculations by Deck [8], obtained on a grid of $4.10^{6}$ cells are also reported for reference. Present results are in excellent agreement with both the reference calculation and the experimental data, in spite of substantially coarser grids. Of course, simulation result for grid 3 better predict the mean flow in the recirculation bubble, especially for the first three profiles of figure 4.

RMS of longitudinal velocity fluctuations are shown in figure 5 . They are in good agreement with the reference ZDES calculation and reasonably close to the experiments, despite the coarseness of grid 1 . Thanks to the non-dissipative numerical scheme, there is no delay in the appearance of Kelvin Helmholtz instabilities in the shear layer: physical perturbations are not damped, and backscatter of energy is fostered. 


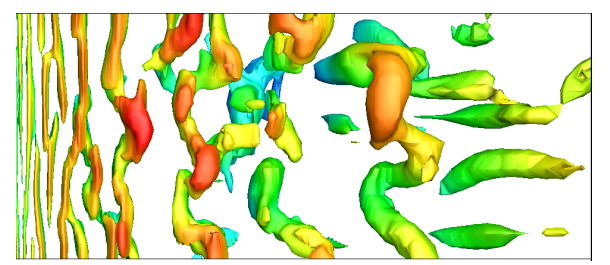

(a) grid 1

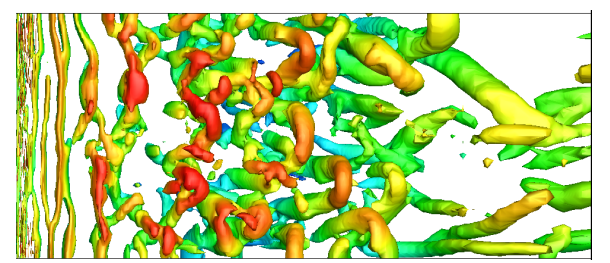

(b) grid 2

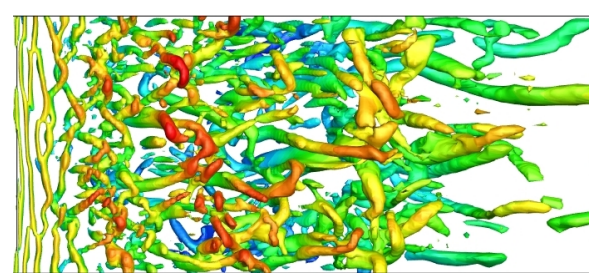

(c) grid 3

Fig. 3 Effect of spanwise grid refinement, iso surface of $\lambda_{2}=-7000$ colored by mean velocity
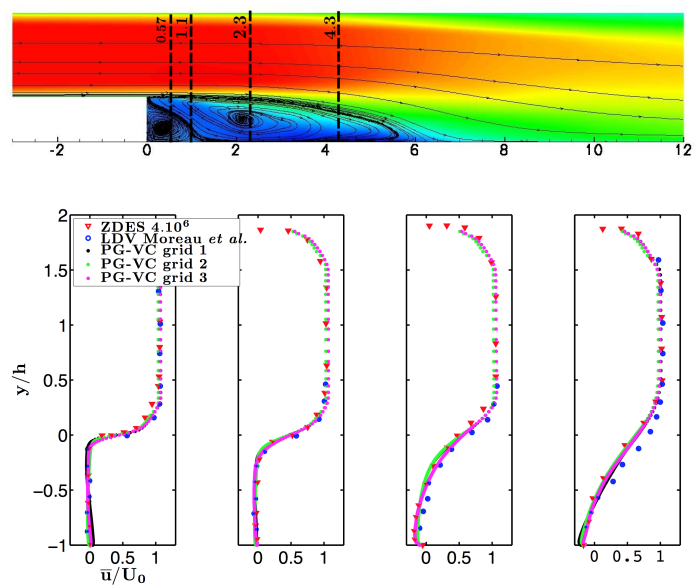

Fig. 4 Mean longitudinal velocity at different locations in the recirculation bubble 

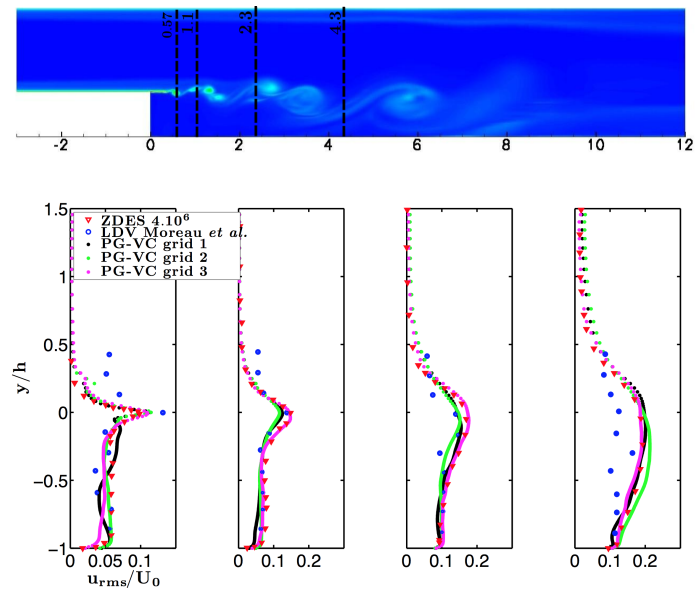

Fig. 5 Longitudinal velocity fluctuations at different locations in the recirculation bubble

\subsection{Axisymmetric backward facing step}

The axisymmetric backward facing step shape was originally designed to be representative of a space launcher vehicle first stage. The test case is a axisymmetric backward facing step experimentally studied by Deprés et al. [12] and Meliga et al. [13]. The geometrical features and measures is_provided on figure 6. The length before the step is chosen so as to obtain a boundary layer thickness of $0.2 \mathrm{D}$ just before the separation point. Present calculation ${ }_{\triangle}$ carried out by using $\mathrm{PG}$ turbulence model coupled with the VC scheme on a grid of $5.7 \times 10^{6}$ cells with average $y^{+}$of the first cell close to the wall of about 50. The $x^{+}$at the beginning of the step is equal to 50. This is rather coarse, but allows keeping computational costs to within an industrially acceptable level. Results are compared to the available experimental data and to $\mathrm{ZDES}^{2}$ calculations [14] [15] with differents grids listed in the tab 2 .

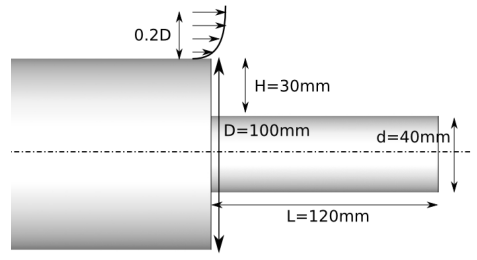

Fig. 6 Schematic of the axisymmetric backward facing step.

\footnotetext{
${ }^{2}$ Zonal Detached Eddy Simulation [8]
} 


\begin{tabular}{|c|c|c|c|}
\hline & ZDES [8] & Present calculation & LDV Experiment[7] \\
\hline $\mathrm{Re}$ & $1.2 \times 10^{6}$ & $1.2 \times 10^{6}$ & $1.2 \times 10^{6}$ \\
\hline $\begin{array}{c}\text { Number } \\
\text { of } \\
\text { cells }\end{array}$ & 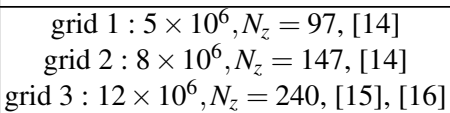 & $\begin{array}{l}5.7 \times 10^{6} \\
N_{z}=120\end{array}$ & $\begin{array}{l}\text { Deprés } \text { et al. [12] } \\
\text { Meliga } \text { et al. [13] }\end{array}$ \\
\hline
\end{tabular}

Table 2 Detail on calculations and experiments. $N_{z}$ is the azimuthal resolution

Figure 7 represents a snapshot of the instantaneous flow field, showing the coherent structures in the recirculation area downstream of the backward facing step. We can see a three-dimensionalization process very similar of the 2D backward facing step flow. Hairpin vortices appear during the pairing mechanism after the separation point and longitudinal vortices are generated downstream of the reattachment point.

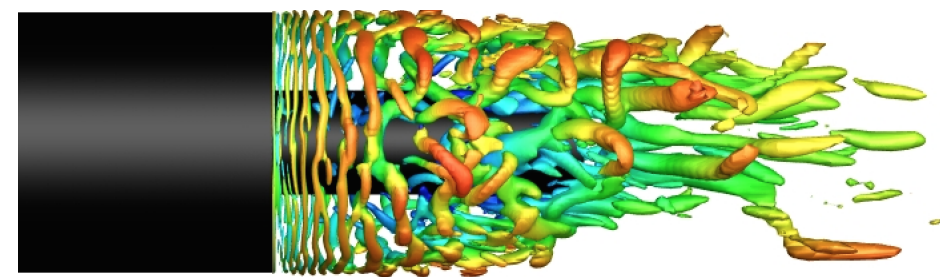

Fig. 7 Coherent structures $=$ isosurface of $\lambda_{2} D / u_{\infty}=10$ colored by instantaneous longitudinal velocity

The mean flow is characterized by a recirculation bubble with reattachment point at $x / D=1.15$. This value is closed to the reattachment point predicted by the ZDES calculation $(x / D=1.1)$ the measurements of Lê [17] $(\mathrm{x} / \mathrm{D}=1.11)$ and Deprés et al $(x / D=1.3)$. Figure 8 shows that the present calculation provides an accurate prediction of pressure coefficient level but the point of minimum $\mathrm{Cp}$ is located downstream

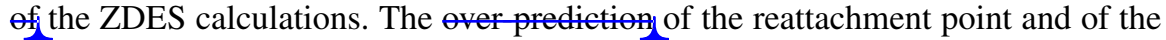
minimum of pressure coefficient is due to the fact that there is a little delay in the triggering of Kelvin Helmholtz instapilities. This likely to be due to the high value of $y^{+}$used in the present calculation-, $\overline{\bar{v}} \mathrm{ch}$ does not allow a good resolution of the shear layer just before the separation point.

Figure 9 shows the spectra of wall pressure fluctuations for several stations along the model. At the beginning of recirculation area $x / D=0.1$ the dominating frequency is $S t r=0.08$; for $x / D=0.6$, the dominating frequency is $S t r=0.2$ and near the point, the dominating frequency corresponds to $S t r=0.58$. Str is the Strouhal number based on $\mathrm{B}_{\mathrm{S}}$. The computed dominating frequencies and their location are in good agreement with the spatial Fourier analysis of Weiss[16] and with the study of Deck and Torigny[14]. The frequency Str $=0.2$ is observed after the calculation of loads on the body by integrating the pressure along the small cylinder since is a shedding like instability. 


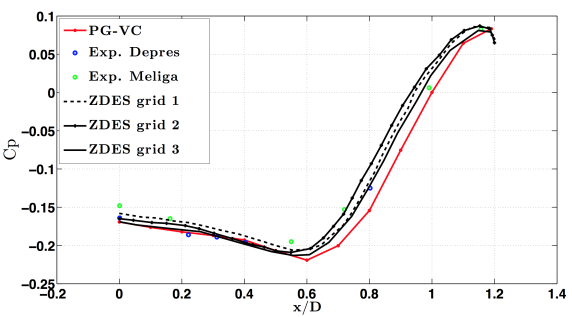

(a) $C p$

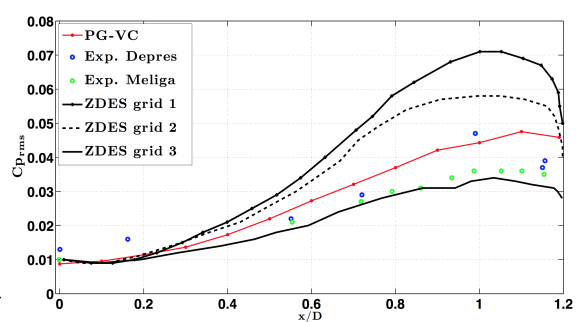

(b) $C p_{r m s}$

Fig. 8 Pressure coefficient on the wall in the recirculation area

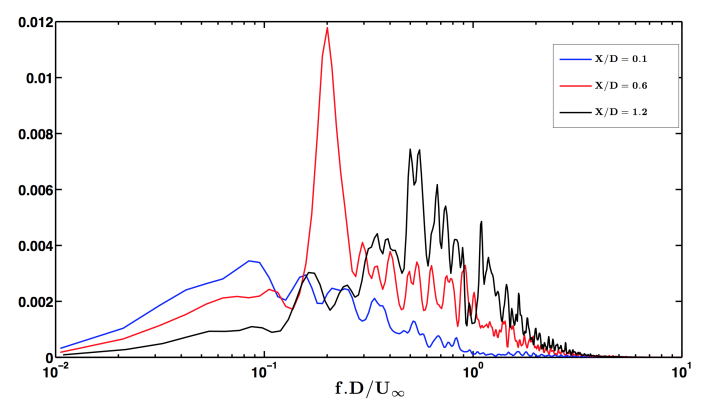

Fig. 9 PSD of pressure on three sensors along the emergency

\section{Conclusion}

We developed a comprehensive HRL strategy combining a self-adaptive hybrid turbulence model and a hybrid high-order unstructured finite volume scheme. The last one is obtained by locally re-centering a third-order upwind scheme in vortexdominated regions, leading to local fourth-order accuracy and to a substantial reduction of the overall numerical dissipation introduced in HRL simulations. This improves the scheme resolvability while ensuring a very good robustness for compressible flows. The HRL model in use is an extension to wall-bounded flows of the one proposed by Perot and Gadebush. This model includes automatic mechanisms for switching from a classical $k-\varepsilon$ model in under-resolved regions and attached boundary layers, to a subgrid model in well-resolved region. It also includes a local antidiffusion mechanism to foster the development of flow instabilities in transition regions from RANS to LES. Numerical results shown for both 2D and axisymmetric flows over backward facing steps are very encouraging and show that the proposed method provides results of reasonable accuracy on relatively coarse grids, leading to an industrially acceptable overall computational cost. 


\section{References}

1. J.Blair Perot and Jason Gadebush. A self-adapting turbulence model for flow simulation at any mesh resolution. Physics of Fluids, 19:1-11, 2007.

2. D. Drikakis. Advances in turbulent flow computations using hight-resolution methods. Progress in Aerospace Sciences, 39:412-424, 2003.

3. F. Haider. Discrétisation en maillage non structuré général et application LES. PhD thesis, UPMC, 2009.

4. G. Pont, P. Cinnella, J-C. Robinet, and Pierre Brenner. Development of numerical schemes for hybrid rans/les modelling in an industrial cfd solver. In AIAA Conference, 2013.

5. T.R.Troutt, B. Scheelke, and T.R. Norman. Organizes structures in a reattaching separated flow filed. Journal of Fluid Mechanics, 143:413-427, 1984.

6. K. Sasaki and M. Kiya. Three-dimensional vortex structure in a leading-edge separation bubble at moderate reynolds numbers. Journal of Fluid Engineering, 113:405-410, 1991.

7. P. Moreau, J. Labb, F.Dupoirieux, and R.Borghi. Experimental and numerical study of a turbulent recirculation zone with combustion. Turbulent Shear Flows, 5:337-346, 1987.

8. S. Deck. Recent improvement in the Zonal Detached Eddy Simulation (ZDES) formulation. Theorical and Computational FLuid Dynamics, 26:523-550, 2011.

9. S.D. Hall, M.Behinia, C.A.J. Fetcher, and G.L. Morrison. Investigation of the secondary corner vortex in a benchmark turbulent backward-facing step using cross-correlation particle imaging velocimetry. Experiments in FLuids, 35:139-151, 2003.

10. D.M. Driver, H. Lee Seegmiller, and J.G. Marvin. Time-dependant behavior of a reattaching shear layer. AIAA Journal, 25:345356, 1985.

11. Hung Le, Parvitz Moin, and John Kim. Direct numerical simulation of turbulent flow over a backward-facing step. Journal of fluid mechanics, 330:349-2374, 1997.

12. D. Deprés, P. Reijasse, and P. Dussauge. Analysis of unsteadiness in after-body transonic flows. AIA Journal, 42:2541, 2004.

13. P.Meliga and P.Reijasse. Unsteady transonic flow behind an axisymmetric afterbody with two boosters. Proceedings of the 25th AIAA Applied Aerodynamics Conference, Paper No.20074564, Miami, 2007.

14. S.Deck and P.Torigny. Unsteadyness of an axisymmetric separating-reattaching flow. Physics of Fluids, 19:065103, 2007.

15. P.E. Weiss, S. Deck, J.C. Robinet, and P. Sagaut. On the dynamics of axisymmetric turbulent separating/reattaching flows. Physics of Fluids, 21, 2009.

16. Pierre-Elie Weiss. Simulation numrique et analyse physique d'un coulement d'arrire-corps axisymtrique et application au contrle des charges latrales. $\mathrm{PhD}$ thesis, Universit Pierre et Marie Curie, 2010.

17. T.H H Lê. Etude expérimentale du couplage entre lécoulement transonique darrière-corps et les charges latérales dans les tuyères propulsives. $\mathrm{PhD}$ thesis, University of Poitiers, 2005. 